\title{
Dime cómo creces y te diré cómo inviertes. El impacto de la I+D, los recursos humanos y los sistemas de innovación en el crecimiento económico: una comparación internacional
}

\author{
Diana Suárez* (D)
}

Florencia Fiorentin ${ }^{* *}$ (D)

Analia Erbes*** (D)

* Universidad Nacional de General Sarmiento, Buenos Aires, Argentina

E-mail: dsuarez@campus.ungs.edu.ar

** Universidad Nacional de General Sarmiento, Buenos Aires, Argentina

E-mail: ffiorentin@campus.ungs.edu.ar

*** Universidad Nacional de General Sarmiento, Buenos Aires, Argentina

E-mail: aerbes@campus.ungs.edu.ar

Recibido en: i6 Septiembre 2019 Versión revisada en: I3 Diciembre 2019 Acceptado: i3 Marzo 2020

\section{Resumen}

Este artículo analiza el impacto de las inversiones en investigación y desarrollo (I+D) y en recursos humanos calificados (RHC) en el crecimiento económico, dado el sistema nacional de innovación (SNI). Pese a la relevancia de estas inversiones, se ha prestado escasa atención a las características de los SNI que las determinan. Los resultados confirman las hipótesis: i) las inversiones en I+D impactan en el crecimiento de los países de ingresos altos y medios; ii) sólo en estos últimos las inversiones en RHC impactan en el crecimiento; iii) ninguno de los tipos de inversiones impacta en los países de ingresos bajos; iv) entre los países de ingresos altos la infraestructura avanzada es el factor explicativo más importante del entorno; en los países de ingreso medio se agrega la infraestructura básica y la equidad y en los países de ingresos bajos solo resulta significativa la infraestructura básica. 
Palabras Clave: Sistemas nacionales de innovación; Investigación y desarrollo; Capacidades, Crecimiento.

\section{Abstract}

This article analyses the impact of investments in research and development (R\&D) and qualified human resources (QHR) on economic growth, subjected to the national innovation system (NIS). Despite the relevance of these investments, little attention has been paid to the characteristics of the NISs that determine them. Results confirm the hypothesis: i) investments in R\&D impact on the growth of high-and medium-income countries; ii) only in the latter investments in QHR impact growth; iii) none of the investments impacts on growth among low-income countries; iv) among high-income countries, advanced infrastructure is the most important explanatory factor of the environment; basic infrastructure and equity are also significant among middle-income countries, and only basic infrastructure is significant in low-income ones.

KeYwords: National innovation systems; Research and development systems; Capabilities, Growth. 


\section{Introducción}

El objetivo de este artículo es analizar el impacto de las inversiones en investigación y desarrollo (I+D) y recursos humanos calificados (RHC) sobre el crecimiento económico, y la medida en que ese impacto (o su ausencia) está determinado por los sistemas nacionales de innovación (SNI). Para ello se parte del consenso que existe en la literatura sobre innovación acerca del rol positivo que tienen las inversiones en I+D y RHC para el crecimiento (p.e.: Castellacci y Natera, 2013; Fagerberg y Srholec, 2008; Kim y Lee, 2015). Se considera también que estas actividades son fuente de generación de conocimiento básico y experimental, cuya aplicación deriva en la generación de innovaciones en el sentido práctico (OCDE/EUROSTAT, 2018) y schumpeteriano del término (SCHUMPETER, 1934, 1942).

Este trabajo se enmarca en el enfoque de los SNI (EDQUIST, 1999; FREEMAN, 1995, LUNDVALL, 1992; NELSON, 1993), dada su capacidad para dar cuenta del proceso innovativo en términos complejos y multidimensionales atendiendo cuestiones que incluyen desde la infraestructura de conocimiento, hasta las reglas de juego y las instituciones que afectan el proceso. Desde esta perspectiva, las actividades de $\mathrm{I}+\mathrm{D}$ y formación de $\mathrm{RHC}$ se consideran elementos clave para explicar las capacidades de absorción e innovación de los países (CHAMINADE; LUNDVALL; HANEEF, 2018). Sin embargo, a pesar del gran desarrollo del enfoque, en especial en la región Latinoamericana (ver ERBES; SUÁREZ, 2019 para una revisión), se ha prestado poca atención a la relación inversa, es decir, el impacto del entorno y las instituciones en la dinámica de realización de actividades de I+D y en sus resultados. Si la innovación es un proceso sistémico, entonces el impacto de las inversiones en la creación y aplicación de conocimiento dependerá del sistema en el que estas actividades tienen lugar.

En este marco, este artículo se propone estudiar las relaciones de causalidad entre I + D y desarrollo. Entender la naturaleza del impacto de la I+D permitiría identificar criterios de política pública respecto de la necesidad de inversiones complementarias. También se propone analizar el impacto de la acumulación de capacidades bajo la forma de RHC, en tanto dimensión complementaria a los esfuerzos en I+D.

El abordaje metodológico implica el estudio econométrico de las inversiones públicas y privadas en actividades de I+D y formación de RHC, y del impacto de estas sobre el producto interno bruto (PIB), sujeto a las características complejas y multidimensionales de los SNI. La evidencia empírica se basa en un macro-panel dinámico construido a partir de la información que publican regularmente Banco 
Mundial, UNESCO, RICyT y EUROSTAT, para el período 2000-2016. Esta base incluye información para un total de 75 países, que representaban más del $97 \%$ del PIB y del 94\% de la población mundial en 2016.

Los resultados muestran que las inversiones en $\mathrm{I}+\mathrm{D}$ tienen impacto positivo en el PIB de los países de altos ingresos y, en menor medida, en los de ingresos medios. Asimismo, sólo en el último grupo de países las inversiones en RHC tienen un efecto positivo en el PIB. Entre los países de ingresos bajos, ni las inversiones en I+D ni los RHC impactan de manera significativa. En lo relativo al entorno, el factor explicativo más importante entre los países desarrollados es la dimensión de infraestructura avanzada, mientras que en los países de desarrollo medio también cobra importancia esta dimensión y se suman la infraestructura básica y la equidad en los ingresos. En los países de bajos ingresos sólo resulta significativo el despliegue de infraestructura básica. Todo esto implica la necesidad de pensar senderos de desarrollo que permitan realizar el catch up tecnológico en los países de ingresos medios y de ingresos bajos atendiendo las especificidades de sus SNI.

El artículo se organiza de la siguiente manera. Luego de esta introducción, en la sección 2 se presenta el marco teórico. En la sección 3 se detalla el abordaje metodológico y la base empírica, y se discuten las hipótesis. En la cuarta sección se analizan los resultados y finalmente, en la sección 5, se proveen algunas conclusiones y propuestas para futuras investigaciones.

\section{Sistemas de innovación, desarrollo y crecimiento económico}

\subsection{Reflexiones conceptuales sobre innovación y desarrollo}

El enfoque de los SNI asume que el cambio tecnológico es central para explicar el crecimiento y el desarrollo económico. La innovación se define como un proceso que se nutre de la creación de capacidades en los agentes (firmas e instituciones), de la infraestructura y de los aparatos científico-tecnológico, cultural y político, entre otros factores del entorno (CHAMINADE; LUNDVALL; HANEEF, 2018). Por lo tanto, el sistema de innovación determina el ritmo y sentido del cambio tecnológico que, a su vez, condiciona las características del proceso de generación y distribución de las cuasi-rentas schumpeterianas.

En la perspectiva dinámica del enfoque se sostiene que existen feedbacks y relaciones no lineales entre los distintos elementos del sistema, en el marco de un fuerte componente path-dependence e irreversibilidad. Cuando las dinámicas de 
retroalimentación son positivas, tienen lugar procesos endógenos de aprendizaje y acumulación de capacidades de forma endógena. El resultado es un proceso virtuoso de crecimiento que se conoce en la literatura como causación acumulativa (MYRDAL, 1974). En sentido inverso, los procesos de subdesarrollo schumpeteriano o destrucción destructiva (REINERT, 1996) se caracterizan por dinámicas de retroalimentación negativa que dan lugar a procesos lock-in donde los sistemas quedan atrapados en estados de reducida acumulación de capacidades y niveles de productividad.

En los estudios sobre innovación en general, y sobre los SNI en particular, las inversiones en conocimiento se presentan como un componente fundamental para el despliegue de procesos de retroalimentación positiva entre acumulación de capacidades, desarrollo de innovaciones y aumento en los niveles de ingreso. Esta dinámica se potencia fuertemente cuando existe un entorno que complementa las capacidades del sector privado, tanto en el sentido schumpeteriano del empresario innovador (SCHUMPETER, 1934), como à la Freeman (1974), en las interacciones entre el empresario y el sistema de ciencia y tecnología. Así, gran parte de las contribuciones desarrolladas desde mediados de los ' 80 s sostienen la relación positiva entre inversiones en conocimiento, medidas en términos de I+D, y crecimiento y desarrollo, los cual se explica por dinámicas complejas de articulación entre oferta y demanda de conocimiento, y otros sectores clave en diferentes niveles de agregación política, sectorial y tecnológica (ARUNDEL et al., 2007; EDQUIST, 2001, 2004; FREEMAN, 1995, 2002; NELSON, 1993).

\subsection{Evidencia empírica e hipótesis}

Desde los inicios del enfoque de los SNI y pari pasu la generación de información estadística para medirlos, han proliferado los análisis que buscan entender la relación entre innovación y crecimiento a nivel internacional. La revisión de esta literatura vinculada con el enfoque de los SNI muestra dos conjuntos de trabajos: aquellos que se enmarcan en una hipótesis de convergencia y los que se encuadran en la de divergencia o especificidades. El marco teórico común de estos trabajos está basado en el enfoque de los SNI donde se asume que el sistema determina el ritmo y sentido del desarrollo tecnológico; se destaca el reconocimiento del rol clave de las actividades de $\mathrm{I}+\mathrm{D}$ como modo de producción de conocimiento científico y tecnológico, y se señala el rol de los recursos humanos calificados como determinantes, á la Penrose (PENROSE, 1959), del rendimiento y desempeño de todos los demás recursos físicos y de conocimiento. 
El primer grupo conceptualiza al desarrollo como un punto de llegada, económico, tecnológico, único y reproducible (ARCHIBUGI; DENNI; FILIPPETTI, 2009; FAGERBERG; MOWERY; VERSPAGEN, 2008). Tomando como referencia un conjunto de indicadores que dan cuenta del grado de desarrollo (p.e.: intensidad tecnológica, estructura productiva y nivel de producto), se postula que los países en desarrollo se encuentran retrasados respecto de los países desarrollados. Luego, se afirma que, en este tipo de países, la inversión sostenida en actividades intensivas en I+D es lo que permitirá alcanzar el nivel de desarrollo objetivo, lo que implica mayores niveles en los indicadores seleccionados. Las innovaciones predominantes deberían ser incrementales en los procesos, mientras que la construcción de capacidades debería enfocarse en la absorción y eventual adaptación de tecnología. Como contrapartida, los países desarrollados serían los responsables de las innovaciones radicales asociadas con la creación de nuevos conocimientos y productos para el mundo.

Una limitación que presentan estos aportes es que no diferencian el destino de las inversiones en I+D ni las características de los países (y sistemas), ya que en la modelización estiman impactos medios significativos para todos los casos que permitirán, en el largo plazo, cerrar la brecha tecnológica y de ingresos entre países. Esto último implica que iguales inputs conducen a iguales outputs, lo cual asume homogeneidad entre países desarrollados y en desarrollo, y entre los que integran cada grupo. Las diferencias entre los países estarán dadas solamente por la velocidad de convergencia al nivel esperado de desarrollo, la cual se asocia con diferentes puntos de partida pero no contempla el rol de diferentes niveles de impacto de las inversiones realizadas, ni la existencia de bloqueos derivados, precisamente, del grado de desarrollo alcanzado. Si bien la I+D es una condición necesaria para que la brecha tecnológica no crezca, no es suficiente para su eliminación (URRACARUIZ; LAGUNA, 2018).

En el segundo grupo de contribuciones, asociado a la hipótesis de divergencia, se asume que el desarrollo requiere de la realización de inversiones específicas para modificar la dinámica del sistema. En tanto cada sistema de innovación es único, no existe un conjunto determinado de dimensiones que conduzca al desarrollo. En este marco, las especificidades nacionales impactan de manera diferencial sobre la relación entre las inversiones en ciencia, tecnología e innovación y el crecimiento económico, por lo que cada país debe encontrar su senda específica.

El abordaje empírico incluye dos tipos de análisis: aquellos basados principalmente en estudios de caso (p.e. los compilados en: Dutrenit y Sutz [2013] para América Latina y Edquist y Hommen [2008] para Asia y Europa), y los que 
buscan identificar patrones de divergencia o clubes de desarrollo (CASTELLACCI; NATERA, 2013; DUTRENIT et al., 2019; LEE, 2013b; LEE; KIM 2009; URRACARUIZ; LAGUNA, 2018). En ambos casos se parte del reconocimiento explícito de diferencias asociadas al nivel de desarrollo, que son causa y consecuencia de la dinámica de los SNI.

Se concluye también que iguales inversiones en I+D y en RHC impactan diferente en cada país o grupo de países. Así, la capacidad de acceder a clubes tecnológicos más complejos, que redundan en mayores niveles de ingreso per cápita, depende de la complejidad en la construcción de capacidades de absorción y de innovación (CASTELLACCI; ARCHIBUGI, 2008), pero también del despliegue de sistemas sociales, políticos, científicos y tecnológicos que provean las instituciones necesarias para acompañar ese desarrollo (DUTRENIT et al., 2019).

Este trabajo se propone contribuir al grupo de estudios que analizan la idea de divergencia, pero desde una perspectiva cuantitativa que también aporte al análisis conceptual de los determinantes del desarrollo. Para ello se testean las siguientes hipótesis:

H1. Las inversiones en actividades de $\mathrm{I}+\mathrm{D}$ tienen impacto positivo y significativo sobre el crecimiento económico;

H2. Las inversiones en RHC tienen impacto positivo y significativo sobre el crecimiento económico;

H3. El nivel de desarrollo de los sistemas nacionales de innovación determina el nivel de impacto de las inversiones en $\mathrm{I}+\mathrm{D}$ y en $\mathrm{RHC}$.

En línea con los estudios sobre convergencia, $\mathrm{H} 1$ y $\mathrm{H} 2$ postulan que, independiente del nivel de desarrollo, las inversiones en I+D y en RHC impactan de forma significativa y positiva en el crecimiento de los países. Estas hipótesis pretenden aportar evidencia empírica actualizada para cuantificar el impacto de dos variables clave para la creación, aplicación y apropiación de conocimiento y la generación de innovaciones. Se espera que a mayores niveles de inversiones en $\mathrm{I}+\mathrm{D}$ y RHC, mayor será su impacto sobre el crecimiento económico.

Contrariamente, en línea con los estudios de divergencia, H3 propone que las características de los SNI son un factor explicativo de la relación entre I+D, RHC y crecimiento. Se sostiene que ambos tipos de inversiones son un elemento con características path-dependence y acumulativas que co-evolucionan en conjunto con los actores e instituciones del SNI. Es esperable que el "rendimiento" de las inversiones en I+D y RHC en sistemas más desarrollados sea mayor que en otros 
menos desarrollados o más débiles, en términos de su composición y relaciones, dados los ya mencionados procesos de causación acumulativa (MYRDAL, 1973).

Asimismo, se espera que las inversiones en I+D y RHC impacten de manera positiva y significativa sobre el crecimiento en las economías de desarrollo medio (p.e. los países latinoamericanos), aunque con menor intensidad que entre los países desarrollados. Dada la "trampa de ingresos medios" (LEE, 2013a), tanto las dimensiones que ubican a estos países cerca de la frontera, como las que suponen bloqueos al desarrollo debieran influir en el impacto de la I+D y los RHC.

Finalmente, es esperable que ambos tipos de inversiones tengan un impacto menor sobre el crecimiento en las economías de ingreso bajo (especialmente en los países africanos de menor nivel de desarrollo relativo). Ello no quiere decir, tal como postula el enfoque de convergencia, que estos países deben obviar las inversiones en $\mathrm{I}+\mathrm{D}$ hasta resolver los problemas del subdesarrollo, sino que, por el contrario, se postula que la $\mathrm{I}+\mathrm{D}$ debe estar articulada con el desarrollo de la infraestructura básica y la satisfacción de demandas de primera necesidad. H3 implica para estos países la necesidad de invertir más que proporcionalmente para generar procesos de leapfrogging en el sendero tecnológico.

\section{Metodología y estrategia de identificación}

\subsection{Base empírica y variables clave}

La metodología implica el análisis econométrico de la evolución de las inversiones públicas y privadas en actividades de I+D y en RHC, y del crecimiento del PIB. La evidencia empírica surge de un macro panel dinámico construido a partir de la información que publican regularmente Banco Mundial, UNESCO, RICyT y EUROSTAT, para el período 2000-2016 (en adelante base mundial). Esta base incluye información sobre variables económicas, desarrollo, la estructura del SNI e inversiones en I+D y RHC. La información está disponible para 75 países que, en conjunto, representaban más del $97 \%$ del PIB y del $94 \%$ de la población mundial en 2016. El período bajo análisis fue segmentado en seis momentos, a partir de los valores promedio: 2000-2002, 2003-2005, 2006-2008, 2009-2011, 2012-2014 y 2015-2016. Esta segmentación y la selección del período maximizan la cantidad de países con información sobre actividades de I+D y RHC. La tabla 1 resume las variables seleccionadas y su tratamiento. 
TABLA 1

Variables, unidad de medida y fuentes de datos

\begin{tabular}{|c|c|c|c|}
\hline & Definición & Unidad de medida & Fuente \\
\hline \multicolumn{4}{|c|}{ Características generales } \\
\hline$\Delta \mathrm{PIB}_{\mathrm{it}}$ & Producto bruto interno & $\begin{array}{l}\text { US\$ } 2005 \text {, pc., tasa de } \\
\text { crecimiento anual. }\end{array}$ & \multirow[t]{3}{*}{ WDI-WB } \\
\hline $\mathrm{Pob}_{\text {it }}$ & Población total & Personas & \\
\hline $\operatorname{Sup}_{\mathrm{i}}$ & Territorio & Kilómetros cuadrados & \\
\hline \multicolumn{4}{|c|}{ Inversiones } \\
\hline$\Delta \mathrm{ID}_{\mathrm{it}}$ & 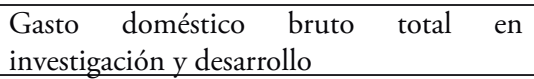 & $\begin{array}{l}\text { US\$ 2005, pc., tasa de } \\
\text { crecimiento anual. }\end{array}$ & \multirow{3}{*}{$\begin{array}{l}\text { UIS - } \\
\text { UNESCO }\end{array}$} \\
\hline$\Delta \mathrm{RHC}_{\mathrm{it}}$ & $\begin{array}{l}\text { Graduados, nivel universitario ( } 4 \text { años o } \\
\text { más) }\end{array}$ & $\begin{array}{l}\text { Número de personas, pc., } \\
\text { tasa de crecimiento anual. }\end{array}$ & \\
\hline Priv/Púb ${ }_{i t}$ & $\begin{array}{l}\text { Gasto doméstico bruto en I+D ejecutado } \\
\text { por el sector privado respecto del público. }\end{array}$ & $\begin{array}{l}\text { Cociente directo, US\$ } \\
\text { 2005, pc. }\end{array}$ & \\
\hline \multicolumn{4}{|c|}{$X:$ Dimensiones SNI } \\
\hline $\mathrm{FC}$ & Formación bruta de capital & US\$2 2005, pc. & \multirow{16}{*}{ WDI-WB } \\
\hline VA_a & Agricultura, valor agregado & US\$ $2005, \mathrm{pc}$. & \\
\hline VA_m & Manufactura, valor agregado & US\$ 2005, pc. & \\
\hline Ex & Exportaciones de bienes y servicios & US\$2005, pc. & \\
\hline Com & Exportaciones de combustibles & $\begin{array}{l}\% \text { total exportaciones de } \\
\text { mercancías }\end{array}$ & \\
\hline HT & $\begin{array}{l}\text { Exportaciones high-tech de bienes y } \\
\text { servicios }\end{array}$ & $\mathrm{U} \$ \mathrm{~S}$ & \\
\hline $\mathrm{Im}$ & Importaciones de bienes y servicios & US\$2 2005, pc. & \\
\hline $\mathrm{CP}$ & Crédito doméstico al sector privado & $\%$ PIB & \\
\hline TI & Tasa de interés real* & $\%$ & \\
\hline Pat & Solicitudes de patentes, residentes & Número & \\
\hline Con_p & $\begin{array}{l}\text { Gasto en consumo final del gobierno } \\
\text { general }\end{array}$ & US\$2 2005, pc. & \\
\hline GINI & $\begin{array}{l}\text { Indice de GINI, estimación Banco } \\
\text { Mundial* }\end{array}$ & Índice & \\
\hline Des & Desempleo total, estimación nacional* & $\%$ fuerza laboral total & \\
\hline Pob & Brecha de pobreza a $\$ 1.90$ por día* & \% línea de pobreza & \\
\hline Prim & Tasa de finalización, nivel primario & \% grupo etario relevante & \\
\hline Sec & Matriculados, nivel secundario & $\%$ neto & \\
\hline Cor & Control de corrupción & Índice & \multirow{6}{*}{ WGI - WB } \\
\hline EG & Efectividad del gobierno & Índice & \\
\hline EP & $\begin{array}{l}\text { Estabilidad política y ausencia de violencia/ } \\
\text { terrorismo }\end{array}$ & Índice & \\
\hline ED & Estado de derecho & Índice & \\
\hline Acc & Voz y rendición de cuentas & Índice & \\
\hline Reg & Calidad regulatoria & Índice & \\
\hline Hom & Homicidios intencionales* & Cada 100.000 personas & \multirow{5}{*}{ WDI-WB } \\
\hline Eng & Acceso a la electricidad & $\%$ población & \\
\hline Int & Usuarios de internet & Cada 100 personas & \\
\hline EV & Esperanza de vida al nacer & Años & \\
\hline Mat & Riesgo de por vida de muerte materna & Personas & \\
\hline \multicolumn{4}{|l|}{ Subindices } \\
\hline$I$ & \multicolumn{3}{|l|}{ País (1-75) } \\
\hline$T$ & \multicolumn{3}{|c|}{ Período de tiempo $(2000-02,2003-05,2006-08,2009-11,2012-14,2015-2016)$} \\
\hline
\end{tabular}

Notas: pc: per capita; WDI-WB: Indicadores World development, base de datos World Bank. UIS-UNESCO: UNESCO Institute for Statistics, base de datos UNESCO. WGI-BM: Indicadores World governance, base de datos World Bank. * Valores inversos $(1 /$ variable). 
El impacto de las inversiones en I+D y RHC será analizado en relación con el PIB per cápita, lo cual es un método habitual en la literatura sobre innovación y desarrollo que permite mejorar la comparabilidad de los resultados. Este indicador se encuentra positivamente asociado tanto con dimensiones que reflejan la calidad de vida de la población (mortalidad, alfabetización, salud, ingresos, etc.), como con aquellas que dan cuenta del crecimiento económico (competitividad, productividad, empleo, etc.). El análisis de la relación entre las tres variables consideradas se realizará a partir de tasas de crecimiento, también en línea con el tratamiento usual en la literatura especializada.

La distinción entre inversiones públicas y privadas en $\mathrm{I}+\mathrm{D}$ responde a una clara diferencia entre los países desarrollados y los países en desarrollo, dado que la relevancia de la participación del sector público en la producción de conocimiento tiene implicancias en las posibilidades de apropiación y explotación. Tal como lo señala la literatura, la mayor parte de la inversión en los países en desarrollo se explica por las inversiones públicas en $\mathrm{I}+\mathrm{D}$, en su mayoría a través de universidades y centros públicos, las cuales llegan a representar, incluso, más de dos tercios del total de esas inversiones (RICYT, 2016). Esto imprime cierta lógica de bien público al conocimiento producido, que se materializa en entornos diferentes a los que posibilitan la destrucción creativa schumpeteriana de donde surge el concepto de innovación (AROCENA; SUTZ, 2016).

Para capturar el impacto de las capacidades se estimó el crecimiento de los graduados universitarios per cápita (RHC), como variable proxy de las capacidades acumuladas de un país. A su vez, se incluyeron controles por región, población y niveles previos de PIB per cápita, como indicativos del punto de partida (FAGERBERG; SRHOLEC, 2008).

\subsection{Caracterización de los sistemas nacionales de innovación}

Complementariamente, y siguiendo la literatura (CASTELLACCI; NATERA, 2013; FAGERBERG; MOWERY; VERSPAGEN, 2008; FAGERBERG; SRHOLEC, $2008)^{1}$ se seleccionaron 30 variables que dan cuenta del SNI (tabla 1) y su relación con el impacto potencial de las inversiones en I+D y RHC sobre el crecimiento del $\mathrm{PIB}^{2}$. Dada la correlación esperadas entre estas variables se realizó un análisis factorial

1 Tanto la metodología de selección como el conjunto final de variables son similares a Fagerberg y Shrolec (2008) y Lee y Kim (2009).

2 Se testearon diferentes conjuntos de variables con resultados similares en términos de los factores explicativos resultantes. 
para identificar la variable latente que explique las diferencias entre sistemas a nivel nacional. Adicionalmente, se utilizaron técnicas de imputación múltiple normal multivariada (LITTLE; RUBIN, 2002), a fin de minimizar el sesgo que se genera por la presencia de mayor cantidad de observaciones faltantes entre los países en desarrollo versus los países desarrollados.

La estimación arrojó seis factores explicativos que reflejan casi el 88\% de las diferencias entre países (tabla 2). El factor más importante representa más del 46\% de la variabilidad entre países y se asocia con la disponibilidad de infraestructura avanzada, en términos del desarrollo productivo e institucional. El segundo factor explica el 12\% de la variabilidad y da cuenta de la disponibilidad de infraestructura básica (educación básica e infraestructura energética). El tercero se refiere al grado de apertura comercial (importaciones y exportaciones) y explica el 10\% de la variabilidad. El cuarto factor combina tamaño del país y su nivel de apropiabilidad tecnológica (países grandes con muchas patentes) y da cuenta del $7 \%$ de la variabilidad. El quinto factor representa simultáneamente el poder adquisitivo de la demanda (inversa del nivel de pobreza) y el desarrollo de infraestructura productiva high-tech de alcance mundial (6\% de la variabilidad). El sexto factor da cuenta de la equidad (6\% de la variabilidad).

TABLA 2

Síntesis del análisis factorial

\begin{tabular}{l|l|c|c}
\hline & \multicolumn{1}{|c|}{ Principales variables } & Autovalor & $\begin{array}{c}\text { Variabilidad } \\
\text { acumulada }\end{array}$ \\
\hline $\begin{array}{l}\text { Infraestructura } \\
\text { avanzada }\end{array}$ & $\begin{array}{l}\text { PBI, formación de capital, crédito } \\
\text { y desarrollo institucional. }\end{array}$ & 9.99885 & 0.462 \\
\hline Infraestructura básica & $\begin{array}{l}\text { Educación primaria y secundaria, } \\
\text { infraestructura eléctrica y de } \\
\text { conectividad. }\end{array}$ & 2.72448 & 0.5879 \\
\hline Apertura comercial & Exportaciones e importaciones & 1.97361 & 0.6791 \\
\hline Apropiabilidad & Patentes, población absoluta & 1.55645 & 0.751 \\
\hline Intensidad tecnológica & Exportaciones high-tech & 1.43072 & 0.8172 \\
\hline Equidad & GINI y mortandad materna & 1.30487 & 0.8774 \\
\hline
\end{tabular}

Notas: panel agregado para todo el período. Observaciones: 429. Valores estandarizados. Variables transformadas para que los valores más altos representen mejores situaciones. Cargas factoriales rotadas. Período 2000-2016.

Fuente: elaboración propia sobre la base mundial. 
Para testear la existencia de impactos diferenciales entre niveles de desarrollo (H3) se agruparon los países en función de sus niveles de ingreso en $\mathrm{t}_{0}$ (2000-2002). El análisis factorial confirmatorio permitió validar la agrupación, y para cada grupo de países existe un vector común de medias asociado a las seis dimensiones identificadas (Tabla 3).

Aunque la agrupación basada en este criterio opaca parte de la heterogeneidad intra-grupos señalada en la sección 2, las combinaciones específicas de las distintas dimensiones no sólo permiten diferenciar niveles de desarrollo, sino también aproximar multidimensionalmente el impacto del entorno. Asimismo, la evidencia muestra que, a los fines de este artículo, la clusterización realizada es válida como criterio de segmentación y hace posible la comparabilidad de los resultados (FAGERBERG; SRHOLEC, 2008; KIM; LEE, 2015; LEE; KIM, 2009). Una alternativa similar a esta agrupación es la utilizada por Urraca-Ruiz y Laguna (2018) en base a Castellacci (2011), en la que se definen "categorías de heterogeneidad" respecto del catch up para cuantificar el impacto diferencial de variables clave del crecimiento.

TABLA 3

Análisis confirmatorio - Factores e ingresos - Cantidad de países (\%)

\begin{tabular}{l|c|c|c}
\hline \multicolumn{1}{c}{$\begin{array}{c}\text { Agrupamiento estimado } \\
\text { según factores }\end{array}$} & \multicolumn{3}{|c}{ Clasificación según ingreso } \\
\cline { 2 - 4 } OCDE & OCDE & Ingreso medio & Ingreso bajo \\
\hline \multirow{2}{*}{ Ingreso medio } & $(94.12)$ & 10 & 0 \\
& 29 & $(5.88)$ & $(0)$ \\
\hline \multirow{2}{*}{ Ingreso bajo } & $(18.83)$ & 125 & 0 \\
& 0 & $(81.17)$ & $10)$ \\
\hline \multirow{2}{*}{ Total } & $(0)$ & 0 & $(100)$ \\
\hline Tasa de error (\%) & 189 & $(0)$ & 105 \\
\hline
\end{tabular}

Notas: panel agregado para todo el período. Observaciones: 429. Distancia de Mahalanobis significativa para todos los grupos. Fuente: elaboración propia sobre la base mundial.

\subsection{Estrategia de estimación}

Dada la naturaleza de la variable dependiente y la necesidad de controlar por los elementos inobservables que se espera que presenten mayor variabilidad entre casos que en el tiempo, se estimó un modelo de efectos fijos, en tanto es esperable que estos inobservables presenten mayor variabilidad entre casos que en el tiempo. Vale 
mencionar que uno de los modelos más utilizados en la literatura es el estimador Arellano-Bond (1991), que permite controlar la endogeneidad que generan las variables fuertemente vinculadas en el tiempo, a partir del despliegue de variables instrumentales. No obstante, estos modelos implican la pérdida significativa de observaciones, dada la instrumentación a partir del retardo de las mismas variables. En este análisis resulta imposible realizar estimaciones diferenciadas por niveles de ingreso. Por este motivo, se optó por utilizar una regresión lineal de efectos fijos, con control de inobservables y condición inicial, siguiendo las soluciones propuestas por Mundlak (1978), Chamberlin (1984) y Wooldridge (2005). Se incluye además el retardo de la variable dependiente, siguiendo con la lógica del estimador ArellanoBond $^{3}$.

Formalmente, el modelo se define como:

$$
\Delta P I B_{i t}=\beta_{1}+\beta_{2} \Delta I D_{i t}+\beta_{3} \Delta R H C_{i t}+\beta_{4} p r i v / p u b_{i t-1}+X_{i t-1}+\mu_{i t}+\epsilon_{i}
$$

Donde $\triangle P I B_{i t} \Delta I D_{i t}$ y $\triangle \mathrm{RHC}_{i t}$ corresponden a las tasas de crecimiento del $\mathrm{PIB}$, las inversiones en $\mathrm{I}+\mathrm{D}$ y $\mathrm{RHC}$, respectivamente, para el país $i$ en el momento t. $\quad p r i v / p u b_{i t-1}$ es el cociente entre las inversiones privadas y públicas; $X_{i t-1}$ corresponde a los 6 factores explicativos resultantes del análisis factorial y $\mu_{i}$ a las variables de control (población, tiempo y niveles previos de PIB) y a los efectos inobservables. La ecuación (1) permite testear las hipótesis $\mathrm{H} 1$ y H2, y la verificación de signo y significatividad de $\beta_{2} y \beta_{3}$ estaría dando cuenta de la magnitud y sentido del impacto de la $\mathrm{I}+\mathrm{D}$ y los $\mathrm{RHC}$ en el crecimiento económico, aproximado a través del PIB.

La estimación de la ecuación (1) para cada uno de los grupos de países permitirá testear H3. Por un lado, los factores explicativos permiten controlar una serie de dimensiones estructurales de los países, que hacen a su desempeño económico. Por el otro, dan cuenta de dimensiones distintas de los SNI, que se verificaron como válidas para dar cuenta de las características de los sistemas. Si existen impactos diferenciales para cada grupo, entonces la magnitud, signo y significatividad de diferirán entre estimaciones.

3 Para testear la robustez de los resultados, se incluye la estimación con el modelo de Arellano-Bond, a partir del cual se obtuvieron resultados similares (Tabla A.1 del anexo). 


\section{Resultados}

\subsection{Patrones de crecimiento y sistemas nacionales de innovación}

La Figura 1. Factores resultantes y grupos de países - Promedios 2003-2016 ilustra las dimensiones identificadas a través del análisis factorial (sección 3.2) que dan cuenta de diferentes características de los SNI. La evidencia permite apreciar patrones diferenciales para cada grupo de países según combinaciones específicas de niveles en cada factor.

\section{FIGURA 1}

Factores resultantes y grupos de países - Promedios 2003-2016

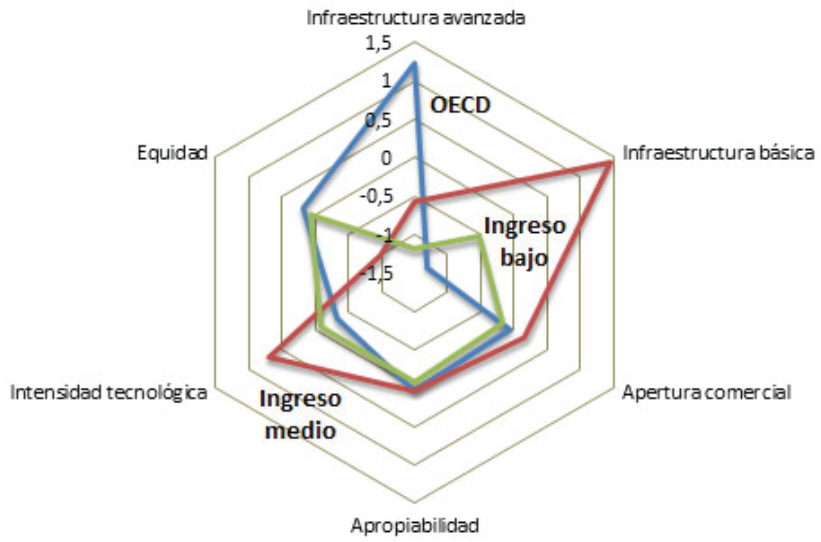

Notas: observaciones 429, valores medios para cada grupo. Muestra agrupada para todo el período. Fuente: elaboración propia sobre base mundial.

Los resultados muestran que los países de la OCDE se caracterizan por un alto desarrollo de la infraestructura avanzada, tanto en lo productivo como en lo institucional. En la cúpula de este grupo se encuentran los países escandinavos (Noruega, Suecia, Dinamarca, Australia y Finlandia) cuyo modelo de desarrollo se destaca además por altos niveles de equidad. A continuación, se encuentran Estados Unidos y Canadá, con un esquema más desigual. Pese a esas diferencias, en ambos tipos de países la infraestructura avanzada es determinante en la relación entre inversiones en $\mathrm{I}+\mathrm{D}$ y crecimiento.

Los países de ingreso medio se destacan por un alto desarrollo de infraestructura básica, con bajos niveles de equidad e intensidad tecnológica. No obstante, en este 
grupo se registran mayores niveles de desvío respecto de los valores medios. Por un lado, los países de América Latina se caracterizan por altos niveles de desigualdad, desequilibrio estructural y deficiencia institucional (DUTRENIT; KATZ, 2005; ERBES; KATZ; SUÁREZ, 2016), al mismo tiempo que se trata de economías principalmente concentradas en la explotación de recursos naturales y de bajo valor agregado (medido en términos de los valores medios del grupo) (BARLETTA; YOGUEL, 2017; DUTRENIT; KATZ, 2005). Por otro lado, los países del sudeste asiático se destacan por ser mayormente exportadores de productos de alto contenido tecnológico (LEE, 2013b; PARK; LEE, 2006). En ambos casos, los niveles medios de desarrollo de infraestructura, pero en contextos de inequidad y baja productividad, son ilustrativos de la trampa de los países de ingresos medios.

Finalmente, en los países de ingresos bajos se observan reducidos niveles en todos los factores. La dimensión de equidad muestra que se trata de países "equitativos" en términos de pobreza, ya que gran parte de la población percibe bajos ingresos. Por ejemplo, Burkina Faso tenía, entre 2015 y 2016, un nivel de GINI similar al de Alemania (32,2 y 31,7, respectivamente), pero mientras que en el primero el PBI per cápita fue de US\$ 654, en el segundo alcanzaba los US\$ 45.630. Existe aquí un limitante claro al crecimiento y desarrollo dado por características propias del círculo de la pobreza que reproducen situaciones de subdesarrollo y dinámicas perversas de causación acumulativa. Aunque su análisis escapa a los objetivos de este trabajo, basta mencionar que en entornos carentes de energía eléctrica o con altas tasas de analfabetismo o contextos sanitarios precarios, las inversiones en $\mathrm{I}+\mathrm{D}$ deberían desempeñar un rol completamente distinto al que se verifica en los países desarrollados o de desarrollo medio.

El análisis comparado entre estos grupos muestra, además, la existencia de una brecha de productividad que explica, tanto la condición de desarrollo de cada uno de los países, como el desafío en materia de catch-up tecnológico. Por ejemplo, el valor agregado en la manufactura entre los países de ingreso medio se ubica en torno al 29\% del valor medio observado para los países de la OCDE. Entre los países de ingreso bajo, esta proporción desciende a $6 \%$. Esto implica que no es posible pensar en un camino tecnológico e institucional único e igual para todos los países.

\subsection{El impacto de las inversiones en I+D y capacidades}

La Erro: Origem da referência não encontradał muestra los resultados de la estimación de la ecuación 1, para el panel total y para cada grupo de países. En primer lugar, 
se verifica $\mathrm{H} 1$ (primera columna). Existe una relación positiva y significativa entre las inversiones en I+D () y el crecimiento del PIB, independientemente del nivel de desarrollo: por cada incremento del $1 \%$ en la tasa de crecimiento de las inversiones en I+D, la tasa de crecimiento del PIB es de $0.035 \%$. De esta forma, los resultados acuerdan con la evidencia y la hipótesis de convergencia planteada por parte de la literatura de los SNI.

En segundo lugar, no se verifica H2. Los resultados muestran una asociación no significativa entre los RHC (y el PIB. Esto sustenta la necesidad de estimarel modelo para cada grupo de países: dada la heterogeneidad de los sistemas, es probable que el efecto difiera entre grupos y que ello funcione como un sesgo del resultado general ${ }^{4}$.

La estimación para cada grupo de países muestra evidencia coincidente con otros aportes presentados en la sección 2 respecto de la hipótesis de especificidades (H3). Los resultados muestran una relación directa entre las características de los SNI y el impacto de la $\mathrm{I}+\mathrm{D}$. El efecto del aumento de la $\mathrm{I}+\mathrm{D}$ en el crecimiento del PIB asciende a $0.12 \%$ entre los países miembros de la OCDE (ingresos altos, segunda columna, tabla 4) y cae a $0.031 \%$ entre los países de ingresos medios, cuando se excluye a China (cuarta columna). Además, entre los países de la OCDE el impacto de la intensidad de la inversión privada es positivo y significativo: por cada dólar de aumento en la inversión privada por encima del incremento de la inversión pública, la tasa de crecimiento del PIB se incrementa en un $0.0127 \%$.

Se excluye a China debido a que, dada la evolución de esta economía durante el período bajo estudio, es esperable que el crecimiento se explique más por dimensiones vinculadas con las instituciones, las relaciones internacionales, las inversiones en infraestructura y el desarrollo productivo, que por la tasa de crecimiento de las inversiones en $\mathrm{I}+\mathrm{D}$ (un fenómeno más reciente en su sendero de crecimiento). No ocurre lo mismo con el crecimiento de los RHC, que impacta positiva y significativamente, lo que es coincidente con el supuesto anterior.

Otro dato interesante es que, entre los países de ingresos medios, el impacto de los RHC sobre el crecimiento es positivo y significativo, mientras que entre los países de la OCDE no es significativo. En los países de ingresos medios, por cada incremento del $1 \%$ en la tasa de crecimiento de los recursos humanos con formación universitaria, la tasa de crecimiento del PIB se incrementa un $0.04 \%$ y $0.027 \%$, con y sin China respectivamente. Es probable que parte de la no significatividad

4 Las pruebas de interacción entre I+D y RHC no arrojaron resultados significativos. Se espera explorar esta relación en futuras investigaciones puesto que desde la teoría se esperaría lo contrario. 
en el impacto de la tasa de crecimiento de los RHC en los países de la OECD se explique por cuestiones de saturación en los niveles absolutos, que además se vincula con menores niveles de crecimiento.

La evidencia sugiere que en los países de ingreso alto capacidades más complejas como las vinculadas con la $\mathrm{I}+\mathrm{D}$, y posiblemente más relacionadas con el sector productivo mediante la inversión privada, son las que explican su crecimiento. En los países de ingreso medio, en cambio, tienen mayor relevancia capacidades de menor complejidad (capacidades productivas vs innovativas, en el sentido de Lee [2013a]). Esto corrobora tanto la dinámica de causación acumulativa o de desarrollo schumpeteriano, como la de trampa de ingresos medios. Entre los países de ingreso medio, la significatividad de la tasa de crecimiento de RHC da cuenta de la necesidad de desarrollar capacidades innovativas, y del mayor horizonte que supone el planeamiento del desarrollo, en tanto son inversiones que toman más tiempo (mínimo los 4/5 años de formación universitaria) versus el incremento en las inversiones en $\mathrm{I}+\mathrm{D}$, cuyo impacto puede materializarse en un plazo relativamente menor.

Por último, con respecto a los países de ingresos bajos (última columna), los impactos tanto de las inversiones en I+D como de los RHC no son significativos. Estos datos son coincidentes con la evidencia que presentan Lee y Kim (2009) respecto de la falta de capacidades productivas elementales como el principal bloqueo al desarrollo de estos países, y van en línea con la postulación de dinámicas lock-in o de subdesarrollo schumpeteriano (REINERT, 1996, 2008). Asimismo, invitan a reflexionar respecto de las inversiones complementarias necesarias para superar los bloqueos o limitaciones al impacto de las inversiones en $\mathrm{I}+\mathrm{D}$.

Lo expuesto se hace más evidente por el resultado negativo y significativo que arroja la dimensión apropiabilidad, principalmente explicada por indicadores de patentes, lo cual podría estar asociado con dos cuestiones. Por un lado, podría suponer que los países de menores ingresos requieren un régimen de circulación de conocimiento más laxo para traccionar procesos de aprendizaje, acumulación de capacidades e innovación orientados a profundizar el crecimiento. Por otro lado, la evidencia para los países latinoamericanos muestra una dinámica innovativa vinculada con mejoras incrementales y procesos de difusión de tecnología que, en muchos casos, no admite la protección del conocimiento a través de patentes (DUTRENIT; KATZ, 2005). 


\begin{tabular}{|c|c|c|c|c|c|c|c|c|c|c|c|c|c|c|c|c|c|c|c|c|}
\hline & & & 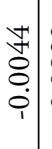 & $\begin{array}{c}0 \\
0 \\
0 \\
0 \\
0 \\
1\end{array}$ & 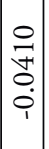 & & $\begin{array}{l}1 \\
\infty \\
0 \\
0 \\
0 \\
1\end{array}$ & 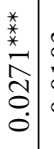 & $\begin{array}{l}n \\
0 \\
0 \\
0\end{array}$ & 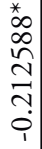 & $\mid \begin{array}{c}0 \\
\stackrel{+}{1} \\
0 \\
0 \\
0\end{array}$ & $\mid \begin{array}{l}0 \\
0 \\
0 \\
0 \\
0 \\
0 \\
1\end{array}$ & 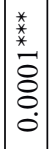 & 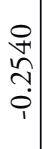 & 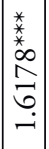 & is & $\mid \begin{array}{l}\infty \\
\vec{f} \\
0 \\
\dot{0}\end{array}$ & $\bar{n}$ & 0 & \\
\hline 氙 & & 岹 & $\begin{array}{c}3 \\
0 \\
0 \\
0\end{array}$ & $\begin{array}{c}\infty \\
n \\
0 \\
0 \\
0\end{array}$ & $\left|\begin{array}{c}\hat{\sigma} \\
\vdots \\
0 \\
0\end{array}\right|$ & & 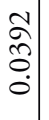 & $\begin{array}{l}+1 \\
8 \\
\vdots \\
\vdots\end{array}$ & 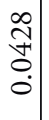 & $\begin{array}{l}\hat{f} \\
\hat{o} \\
\dot{0}\end{array}$ & $\left|\begin{array}{c}- \\
0 \\
0 \\
0 \\
0\end{array}\right|$ & $\mid \begin{array}{c}2 \\
\overline{8} \\
0 \\
0\end{array}$ & $\mid \begin{array}{l}0 \\
8 \\
0 \\
0 \\
0\end{array}$ & $\mid \begin{array}{l}\overline{\hat{\sigma}} \\
0 \\
\dot{0}\end{array}$ & $\mid \begin{array}{l}\stackrel{+}{n} \\
\stackrel{n}{0} \\
0\end{array}$ & & & & & \\
\hline 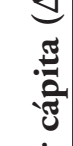 & & نे & $\begin{array}{l}\stackrel{*}{m} \\
\tilde{0} \\
\stackrel{0}{0}\end{array}$ & \begin{tabular}{c}
$*$ \\
\multirow{N}{N}{} \\
0 \\
0 \\
0
\end{tabular} & $\mid$\begin{tabular}{c}
\multirow{1}{*}{} \\
0 \\
8 \\
0 \\
0
\end{tabular} & & $\begin{array}{l}\tilde{n} \\
0 \\
0 \\
0\end{array}$ & \begin{tabular}{l|l}
$n$ \\
$\vdots$ \\
$\vdots$ \\
$\vdots$ \\
1
\end{tabular} & 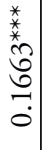 & 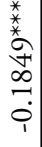 & \begin{tabular}{c}
\multirow{1}{*}{} \\
$\grave{8}$ \\
0 \\
$\dot{0}$
\end{tabular} & 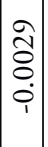 & $\begin{array}{l}-1 \\
8 \\
0 \\
\vdots \\
0\end{array}$ & 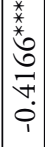 & 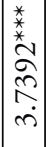 & is & 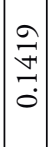 & $\diamond$ & $\stackrel{\sim}{\sim}$ & \\
\hline$\frac{\theta}{\tilde{\theta}}$ & & 5 & $\begin{array}{l}\overrightarrow{\tilde{J}} \\
0 \\
\dot{0}\end{array}$ & 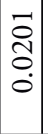 & $\mid \begin{array}{c}0 \\
\infty \\
0 \\
0 \\
0\end{array}$ & & 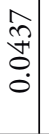 & $\begin{array}{l}+ \\
8 \\
\vdots \\
\dot{0}\end{array}$ & $\begin{array}{l}0 \\
\tilde{\delta} \\
0 \\
0 \\
0\end{array}$ & 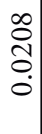 & $\left|\begin{array}{l}0 \\
0 \\
\vdots \\
0 \\
0\end{array}\right|$ & 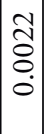 & $\mid \begin{array}{l}8 \\
0 \\
\vdots \\
0 \\
0\end{array}$ & $\mid \begin{array}{l}\vec{F} \\
\hat{a} \\
\dot{0}\end{array}$ & $\mid \begin{array}{l}\infty \\
2 \\
\infty \\
\hat{0} \\
\dot{0}\end{array}$ & & & & & \\
\hline : & 总 & डे & 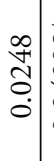 & 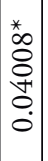 & $\left|\begin{array}{l}m \\
\cdots \\
0 \\
0 \\
1\end{array}\right|$ & & 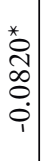 & $\begin{array}{l}+1 \\
0 \\
0 \\
0 \\
1\end{array}$ & 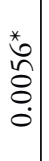 & $\begin{array}{l}\text { * } \\
0 \\
0 \\
0 \\
0 \\
0 \\
1\end{array}$ & $\mid \begin{array}{c}0 \\
0 \\
0 \\
0 \\
0 \\
1\end{array}$ & $\mid \begin{array}{l}1 \\
0 \\
0 \\
0 \\
0 \\
0\end{array}$ & $\mid$\begin{tabular}{l|}
$*$ \\
\multirow{*}{*}{} \\
$\vdots$ \\
8 \\
$\vdots$ \\
0 \\
0
\end{tabular} & 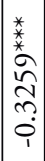 & $\begin{array}{c}\bar{\Sigma} \\
\vdots \\
i \\
i\end{array}$ & is & $\mid \begin{array}{l}\vec{n} \\
\infty \\
\overrightarrow{0} \\
0\end{array}$ & $\stackrel{-}{ }$ & $\approx$ & 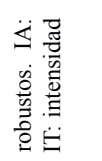 \\
\hline $\begin{array}{l}\ddot{\partial} \\
\frac{\partial}{v} \\
\dot{\vec{z}}\end{array}$ & 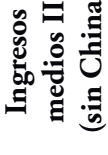 & 5) & $\begin{array}{l}\infty \\
\vec{\forall} \\
0 \\
0\end{array}$ & $\begin{array}{l}-+ \\
\tilde{1} \\
0 \\
0\end{array}$ & $\mid \begin{array}{c}-1 \\
\vdots \\
0 \\
0\end{array}$ & & $\begin{array}{l}\infty \\
\vec{\forall} \\
0 \\
\dot{0}\end{array}$ & $\begin{array}{l}\overrightarrow{8} \\
\vdots \\
\vdots\end{array}$ & $\begin{array}{c}\tilde{0} \\
0 \\
0 \\
0\end{array}$ & $\begin{array}{l}\tilde{\hat{\jmath}} \\
\hat{\sigma} \\
\dot{0}\end{array}$ & 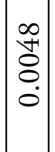 & $\mid \begin{array}{c}1 \\
0 \\
0 \\
0 \\
0\end{array}$ & $\begin{array}{l}0 \\
\vdots \\
\vdots \\
0 \\
\vdots\end{array}$ & $\mid \begin{array}{l}0 \\
+ \\
0 \\
0 \\
0\end{array}$ & $\begin{array}{l} \\
0 \\
0 \\
\infty \\
0 \\
0\end{array}$ & & & & & 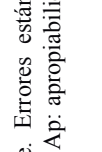 \\
\hline 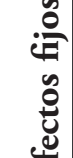 & 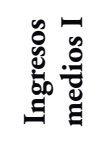 & نे & $\begin{array}{l}\stackrel{*}{*} \\
\stackrel{*}{2} \\
\Xi \\
\vdots \\
0\end{array}$ & $\begin{array}{l}N \\
\hat{O} \\
8 \\
0 \\
0\end{array}$ & $\mid \begin{array}{c}* \\
\stackrel{*}{*} \\
\stackrel{0}{0} \\
0 \\
0\end{array}$ & & $\begin{array}{l}\exists \\
\Xi \\
0 \\
0\end{array}$ & $\begin{array}{l}5 \\
\vdots \\
\vdots \\
\dot{0}\end{array}$ & $\begin{array}{c}\infty \\
\overrightarrow{0} \\
0 \\
0\end{array}$ & 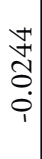 & $\mid \begin{array}{l}\overrightarrow{1} \\
\stackrel{0}{0} \\
\dot{0}\end{array}$ & $\mid \begin{array}{c}8 \\
8 \\
0 \\
0 \\
0\end{array}$ & $\begin{array}{l}\vec{b} \\
\vdots \\
0 \\
0\end{array}$ & $\mid \begin{array}{l}* \\
* \\
* \\
\hat{*} \\
\tilde{N} \\
\tilde{n} \\
\vdots \\
1\end{array}$ & 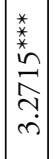 & is & స్ & 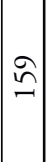 & $\stackrel{+}{m}$ & 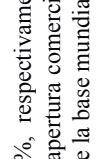 \\
\hline $\begin{array}{c}1 \\
0 \\
0\end{array}$ & $\begin{array}{l}\text { Оి } \\
\text { 둉 }\end{array}$ & के & $\begin{array}{c}R \\
0 \\
0 \\
0\end{array}$ & $\begin{array}{l}\stackrel{0}{n} \\
0 \\
0 \\
0\end{array}$ & 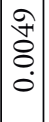 & & $\begin{array}{l}0 \\
\vec{\Delta} \\
0 \\
0\end{array}$ & $\begin{array}{l}c \\
\vdots \\
\vdots \\
\vdots \\
\vdots\end{array}$ & $\begin{array}{c}\infty \\
\vdots \\
0 \\
0\end{array}$ & $\begin{array}{l}\hat{n} \\
0 \\
0 \\
0\end{array}$ & $\mid \begin{array}{l}0 \\
2 \\
8 \\
0 \\
0\end{array}$ & $\mid \begin{array}{l}0 \\
8 \\
0 \\
0\end{array}$ & $\begin{array}{l}0 \\
\vdots \\
\vdots \\
0 \\
\dot{0}\end{array}$ & $\mid \begin{array}{l}n \\
n \\
0 \\
0 \\
0 \\
0\end{array}$ & $\mid \begin{array}{l}N \\
\tilde{o} \\
+ \\
0 \\
0\end{array}$ & & & & & 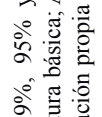 \\
\hline 苞 & 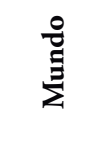 & نे & \begin{tabular}{l}
$*$ \\
\multirow{2}{*}{} \\
$\tilde{2}$ \\
0 \\
0 \\
0
\end{tabular} & $\begin{array}{l}n \\
\hat{o} \\
0 \\
0\end{array}$ & $\mid \begin{array}{l}n \\
\tilde{\delta} \\
0 \\
0\end{array}$ & & 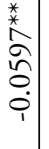 & \begin{tabular}{l|l}
8 \\
$\vdots$ \\
$\vdots$ \\
$\vdots$
\end{tabular} & 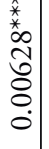 & 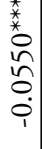 & $\mid \begin{array}{l}n \\
\hat{\delta} \\
\vdots \\
0\end{array}$ & \begin{tabular}{|l|}
8 \\
8 \\
8 \\
0 \\
0
\end{tabular} & 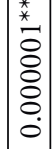 & 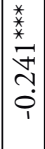 & $\mid$\begin{tabular}{c}
$*$ \\
\multirow{*}{*}{} \\
$\hat{n}$ \\
$\underline{6}$ \\
$\stackrel{-}{i}$
\end{tabular} & is & 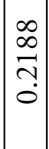 & $\begin{array}{l}0 \\
m \\
n\end{array}$ & $\curvearrowleft$ & 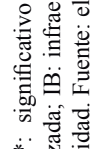 \\
\hline & & & $\stackrel{\star}{\triangleleft}$ & 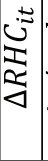 & 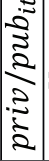 & 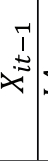 & 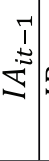 & $\begin{array}{c}1 \\
\vdots \\
0 \\
0\end{array}$ & 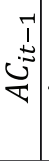 & 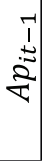 & 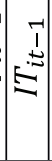 & $\left|\begin{array}{c}1 \\
1 \\
\vdots \\
0 \\
01\end{array}\right|$ & 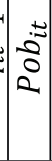 & $\frac{\vdots}{20}$ & 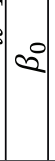 & ت & No & $z$ & $\dot{\overrightarrow{0}}$ & 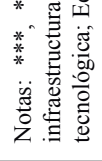 \\
\hline
\end{tabular}


Por lo tanto, estos resultados ponen de manifiesto la necesidad de profundizar en los determinantes de la $\mathrm{I}+\mathrm{D}$ y en cómo estas inversiones son a la vez causa y consecuencia de las características del entorno donde se desarrollan.

\section{Conclusiones}

Este artículo se propuso analizar el impacto de las inversiones en investigación y desarrollo (I+D) y recursos humanos calificados (RHC) en el crecimiento económico, y evaluar la medida en que ese impacto está determinado por las características de los sistemas nacionales de innovación (SNI). El marco teórico adoptado es el de los SNI, por ser un enfoque que comprende la relevancia del cambio tecnológico y de las capacidades, en toda su complejidad, para los procesos de aprendizaje y desarrollo de los países.

A partir de este enfoque se entiende que las inversiones en $\mathrm{I}+\mathrm{D}$ y RHC impactan de manera iterativa, acumulativa y sistémica, y que ello depende de la historia social, cultural, política, etc.- de los sistemas y de los senderos de crecimiento y acumulación de capacidades previos. Por lo tanto, se asume que la relación entre las inversiones en I+D y RHC y el crecimiento no se manifiesta de igual manera en todos los países. Más aun, existen especificidades de los SNI que regulan esa relación, e incluso pueden actuar como bloqueos al crecimiento y desarrollo.

Se plantearon tres hipótesis que sugieren que las inversiones en I+D y RHC impactan positiva y significativamente sobre el crecimiento de los países. No obstante, dicho impacto varía entre distintos grupos de países, y ello se encuentra determinado por las características estructurales de sus SNI. Los resultados permitieron comprobar las hipótesis presentadas. En los países de ingreso alto, las inversiones en $\mathrm{I}+\mathrm{D}$, y en particular las inversiones privadas, impactan de forma positiva y significativa en su crecimiento, en línea con los procesos de causación acumulativa planteados por Myrdal (1974). Adicionalmente, un factor explicativo de sus SNI son las características de la infraestructura avanzada, tanto en lo productivo como en lo institucional.

En segundo lugar, en los países de ingreso medio las inversiones en $\mathrm{I}+\mathrm{D}$ también generan efectos positivos en su crecimiento, pero en menor medida que en el caso de los países desarrollados. Asimismo, y a diferencia de los primeros, en este grupo también tiene impacto positivo y significativo la inversión en RHC. Se verifica así la trampa de ingresos medios (LEE, 2013b). A su vez, tanto las dimensiones de infraestructura básica y equidad, como de infraestructura avanzada resultan factores explicativos del crecimiento. 
Por último, en los países de ingreso bajo no se verifica relación significativa entre crecimiento e inversiones en $\mathrm{I}+\mathrm{D}$ y $\mathrm{RHC}$, sino que el factor explicativo relevante es la infraestructura básica. En este caso, los resultados concuerdan con la literatura que identifica procesos de subdesarrollo schumpeteriano (REINERT, 1996). Adicionalmente, estos resultados se encuentran en línea con la evidencia hallada por Castellacci y Natera (2013) y otros estudios enmarcados en el grupo de los clubes de convergencia, que corroboran el impacto diferencial de las inversiones en I+D y RHC sobre el crecimiento y desarrollo.

Las limitaciones del trabajo se relacionan con el sesgo que presentan las bases de datos internacionales hacia los países de mayor desarrollo, ya que la mayor disponibilidad de datos sobre estas economías sobreestima los resultados medios. Otra limitación se vincula con la cantidad de indicadores disponibles. Las inversiones en I+D recién se compilan sistemáticamente y para un grupo amplio de países desde principios de 2000. Como consecuencia, la información acumulada no alcanza a las dos décadas, lo cual representa un horizonte temporal acotado para evaluar los fenómenos analizados en esta investigación. Esto limita el análisis en función de las ventanas de tiempo posibles entre la realización de inversiones y su impacto y restringe las opciones econométricas para testear sus efectos.

No obstante estas limitaciones, los resultados iniciales de esta investigación invitan a reflexionar sobre cómo pueden superar su condición-condena los países de ingresos bajos y de ingresos medios, y el rol de la I+D y los RHC en ese proceso. En ese sentido, es evidente que deben avanzar en mejoras institucionales y de desarrollo de infraestructura, que permitan desencadenar dinámicas positivas vinculadas con sus propias características, mediante inversiones más que proporcionales en conocimiento. Estar lejos de la frontera implica para los países el desafío simultáneo de cerrar la brecha tecnológica y avanzar en un sendero de desarrollo, pero atendiendo a las características de sus propias especificidades y, más especialmente, al sendero de desarrollo deseado. Académicos y hacedores de política deben asumir el desafío de pensar en términos de la multidimensionalidad que caracteriza al crecimiento, el desarrollo y la CTI lo cual, a su vez, implica pensar que un aumento en el PBI no puede entenderse disociado de cuestiones elementales como la equidad e inclusión social. 


\section{ANEXO}

TABLA A. 1

Análisis de robustez - Var. dep: Crecimiento del PIB

\begin{tabular}{|c|c|c|c|c|c|c|c|}
\hline & \multicolumn{2}{|c|}{ OLS agregado } & \multicolumn{2}{|c|}{ Efectos fijos (I) } & \multicolumn{2}{|c|}{ Efectos fijos (II) } & \multirow{2}{*}{$\begin{array}{c}\begin{array}{c}\text { Arellano- } \\
\text { Bond }\end{array} \\
\text { Coef }\end{array}$} \\
\hline & Coef & $S E$ & Coef & $S E$ & Coef & $S E$ & \\
\hline$\Delta I D_{i t}$ & $0.0562^{* *}$ & 0.0221 & $0.0338^{* *}$ & 0.0172 & $0.0356^{* *}$ & 0.0170 & $0.0758^{*}$ \\
\hline$\Delta R H C_{i t}$ & -0.0152 & 0.0177 & 0.0110 & 0.0159 & 0.0075 & 0.0156 & 0.0047 \\
\hline priv/pub $b_{i t-1}$ & 0.0026 & 0.0040 & 0.0027 & 0.0044 & 0.0055 & 0.0049 & 0.0025 \\
\hline \multicolumn{8}{|l|}{$X_{i t-1}$} \\
\hline$I A_{i t-1}$ & -0.0059 & 0.0057 & & & $-0.060^{* * *}$ & 0.0216 & $-0.148^{* * *}$ \\
\hline$I B_{i t-1}$ & -0.0002 & 0.0001 & & & 0.0000 & 0.0002 & 0.0001 \\
\hline$A C_{i t-1}$ & 0.0011 & 0.0015 & & & $0.0063^{* * *}$ & 0.0018 & -0.0034 \\
\hline$A p_{i t-1}$ & $-0.0118^{* * *}$ & 0.0043 & & & $-0.055^{* * *}$ & 0.0157 & 0.0102 \\
\hline$I T_{i t-1}$ & 0.0004 & 0.0021 & & & 0.0035 & 0.0056 & -0.0111 \\
\hline$E q_{i t-1}$ & -0.0002 & 0.0002 & & & 0.0000 & 0.0001 & 0.0008 \\
\hline$P o b_{i t-1}$ & $0.0001^{* * *}$ & 0.0000 & $0.0001^{* * *}$ & 0.0000 & $0.0001^{* * *}$ & 0.0000 & 0.0000 \\
\hline$\Delta P I B_{i t-1}$ & $-0.0224^{* * *}$ & 0.0071 & $-0.265^{* * *}$ & 0.0440 & $-0.241^{\text {*** }}$ & 0.0455 & 0.0000 \\
\hline$\beta_{0}$ & $0.2954^{* * *}$ & 0.0645 & $2.4151^{* * *}$ & 0.3860 & 2.1657 & 0.4052 & $0.2854^{*}$ \\
\hline$t_{i}$ & Sí & & Sí & & Sí & & Sí \\
\hline Ingreso $_{i}$ & & & & & & & Sí \\
\hline$R^{2}$ & 0.4883 & & 0.2126 & & 0.2188 & & \\
\hline$N$ & 310 & & 310 & & 310 & & 176 \\
\hline Obs. & & & 75 & & 75 & & 69 \\
\hline \multicolumn{7}{|c|}{$\begin{array}{l}\text { Notas: }{ }^{* * *},{ }^{* *} y^{*} \text { : significativo al } 99 \%, 95 \% \text { y } 90 \% \text {, respectivamente. IA: infraestructura } \\
\text { avanzada; IB: infraestructura básica; AC: apertura comercial; Ap: apropiabilidad; IT: } \\
\text { intensidad tecnológica; Eq: equidad. Errores estándar robustos. Fuente: elaboración } \\
\text { propia sobre la base mundial. }\end{array}$} & \\
\hline
\end{tabular}

\section{Agradecimientos}

Las autoras agradecen a los dos revisores anónimos por sus comentarios. Agradecemos también los comentarios recibidos en las conferencias de la red Lalics, la conferencia por el 50mo. Aniversario del SPRU y la II Conferencia sobre Planificación del Desarrollo Julio H. Olivera.

\section{Referencias}

ARCHIBUGI, D.; DENNI, M.; FILIPPETTI, A. The technological capabilities of nations: The state of the art of the synthetic indicators. Technological Forecasting and Social Change, v. 76, n. 7, p. 917-931, 2009. 
ARELLANO, M.; BOND, S. Some Tests of Specification for Panel Data: Monte Carlo Evidence and an Application to Employment Equations. The Review of Economic Studies, v. 58, n. 2, p. 277, 1 Apr. 1991.

AROCENA, R.; SUTZ, J. Innovación y sistemas nacionales de innovación en procesos de desarrollo. In: ERBES, A.; SUAREZ, D. (ed.). Repensando el desarrollo latinoamericano. Una discusión desde los sistemas de innovación. Buenos Aires: UNGS, 2016.

ARUNDEL, A.; LORENZ, E.; LUNDVALL, B.-Å.; VALEYRE, A. How Europe’s economies learn: a comparison of work organization and innovation mode for the EU-15. Industrial and Corporate Change, v. 16, n. 6, p. 1175-1210, 2007.

BARLETTA, F.; YOGUEL, G. ¿De qué hablamos cuando hablamos de cambio estructural? In: ABELES, M.; CIMOLI, M.; LAVARELLO, P. (ed.). Manufactura y cambio estructural: aportes para pensar la política industrial en la Argentina. [s.1.] CEPEAL, 2017. p. 27-54.

CASTELLACCI, F. Closing the Technology Gap? Review of Development Economics, v. 15, n. 1, p. 180-197, 2011.

CASTELLACCI, F.; ARCHIBUGI, D. The technology clubs: The distribution of knowledge across nations. Research Policy, v. 37, n. 10, p. 1659-1673, 2008.

CASTELLACCI, F.; NATERA, J.M. The dynamics of national innovation systems: A panel cointegration analysis of the coevolution between innovative capability and absorptive capacity. Research Policy, v. 42, n. 3, p. 579-594, 2013.

CHAMBERLAIN, G. Panel Data. In: GRILICHES, Z.; INTRILIGATOR, M. (ed.). Handbook of Econometrics. Amsterdam: North Holland, 1984. v. 2. p. 1247-1318.

CHAMINADE, C.; LUNDVALL, B.-Å.; HANEEF, S. Advanced introduction to national innovation systems. [s.1.] Elgar, 2018.

DUTRENIT, G.; KATZ, J. Innovation, growth and development in Latin-America: Stylized facts and a policy agenda. Innovation: Management, Policy \& Practice, v. 7, n. 2-3, p. 105-130, 2005.

DUTRÉNIT, G.; NATERA, J.M.; PUCHET, M.; VERA-CRUZ, A. Development profiles and accumulation of technological capabilities in Latin America. Technological Forecasting and Social Change, v. 145, p. 396-412, 2019.

DUTRENIT, G.; SUTZ, J. Sistemas de innovación para un desarrollo inclusivo. Mexico: Foro consultivo científico y tecnológico - Lalics, 2013.

EDQUIST, C. Systems of Innovation. Technologies, Institutions and Organizations. London: Pinter, 1997. 
EDQUIST, C. Systems of Innovation for Development (SID). In: Competitiveness, Innovation and Learning: Analytical Framework. for the UNIDO World Industrial Development Report (WIDR), [s.l.], 2001. (Background Paper for Chapter I).

EDQUIST, C. Systems of innovation: perspectives and challenges. In: FAGERBERG, J.; MOWERY, D.; NELSON, R. (ed.). The Oxford Handbook of Innovation. USA: Oxford University Press, 2004.

EDQUIST, C.; HOMMEN, L. Comparing national systems of innovation in Asia and Europe: theory and comparative framework. In: EDQUIST, C.; HOMMEN, L. (ed.). Small Country Innovation Systems: Globalization, Change and Policy in Asia and Europe. Cheltenham: Edward Elgar publishing, 2008. Chapter 1. p. 1-30.

ERBES, A.; KATZ, J.; SUAREZ, D. Aportes latinoamericanos en la construcción del enfoque de SNI. El énfasis en el desarrollo. In: ERBES, A.; SUAREZ, D. (ed.). Repensando el desarrollo latinoamericano. Una discusión desde los sistemas de innovación. Buenos Aires: UNGS, 2016.

ERBES, A.; SUAREZ, D. Sistemas nacionales de innovación: antecedentes y debates. In: SUAREZ, D.; ERBES, A.; BARLETTA, F. (ed.). Teoría de la innovación: evolución, tendencias y desafíos. Herramientas conceptuales para la enseńanza y el aprendizaje. Buenos Aires y Madrid: UNGS-UCM, 2019.

FAGERBERG, J.; MOWERY, D.; VERSPAGEN, B. Innovation-systems, path-dependency and policy: The coevolution of science, technology and innovation policy and industrial structure in a small, resource-based economy, 2008. (Working Papers on Innovation Studies, Centre for Technology, Innovation and Culture, University of Oslo).

FAGERBERG, J.; SRHOLEC, M. National innovation systems, capabilities and economic development. Research Policy, v. 37, n. 9, p. 1417-1435, 2008.

FREEMAN, C. The Economics of Industrial Innovation. Harmondsworth, Middlesex: Penguin Books, 1974.

FREEMAN, C. The 'National System of Innovation' in historical perspective. Cambridge Journal of Economics, v. 19, n. 1, p. 5-24, 1995.

FREEMAN, C. Continental, national and sub-national innovation systems-complementarity and economic growth. Research Policy, v. 31, n. 2, p. 191-211, 2002.

JIMÉNEZ, J.P. Desigualdad, concentración del ingreso y tributación sobre las altas rentas en América Latina. [s.1.] Comisión Económica para América Latina y el Caribe, 2015.

KIM, Y.K.; LEE, K. Different Impacts of Scientific and Technological Knowledge on Economic Growth: Contrasting Science and Technology Policy in East Asia and Latin America. Asian Economic Policy Review, v. 10, n. 1, p. 43-66, 2015. 
LEE, K. Capability Failure and Industrial Policy to Move beyond the Middle-Income Trap: From Trade-based to Technology-based Specialization. In: STIGLITZ J.E., LIN, J.Y. (ed.). The Industrial Policy Revolution I. London: Palgrave Macmillan, 2013a. p. 244-272.

LEE, K. Schumpeterian analysis of economic catch-up: Knowledge, path-creation, and the middle-income trap. [s.l.] Cambridge University Press, 2013b.

LEE, K.; KIM, Y.K. Both Institutions and Policies Matter but Differently for Different Income Groups of Countries: Determinants of Long-Run Economic Growth Revisited. World Development, v. 37, n. 3, p. 533-549, 2009.

LITTLE, R.; RUBIN, D. Statistical analysis with missing data. New Jersey. 2002.

LUNDVALL, B.-Å. National System of Innovation: Towards a Theory of Innovation and Interactive Learning. London Pinter, 1992.

MUNDLAK, Y. On the pooling of time series and cross section data. Econometrica, v. 46, p. 69-85, 1978.

MYRDAL, G. Equity and growth. World Development, v. 1, n. 11, p. 43-47, 1973.

MYRDAL, G. What Is Development? Journal of Economic Issues, v. 8, n. 4, p. 729-736, 1974.

NELSON, R. National Innovation Systems: A Comparative Analysis. Oxford: Oxford University Press, 1993.

OCDE/EUROSTAT. Oslo Manual 2018: Guidelines for Collecting, Reporting and Using Data on Innovation, 4th Edition, The Measurement of Scientific, Technological and Innovation Activities. Paris/Eurostat, Luxembourg: OECD, 2018.

PARK, K.-H.; LEE, K. Linking the technological regime to the technological catch-up: analyzing Korea and Taiwan using the US patent data. Industrial and Corporate Change, v. 15, n. 4, p. 715-753, 1 Aug. 2006.

PENROSE, E. The theory of the growth of the firm. Oxford: Oxford University Press, 1959.

REINERT, E. The role of technology in the creation of rich and poor nations: underdevelopment in a Schumpeterian system. In: ALDCROFT; CATTERALL (ed.). Rich nations-poor nations. UK: Elgar, 1996. p. 431-456.

REINERT, E. How rich countries got rich and why poor countries stay poor. [s.l.] Public Affairs, 2008.

RICYT. Indicadores de Ciencia y Tecnología, 2016. Available on: www.ricyt.org. Accessed on:_16 set.2019.

SCHUMPETER, J. sThe theory of economic development. Cambridge: Harvard University Press, 1934. (First edition, 1912). 
SCHUMPETER, J. Capitalism, socialism, and democracy. London: Harper and Brothers, 1942.

SZTULWARK, S. Especialización Productiva y Subdesarrollo en el Paradigma Informacional. Una Aproximación a Partir del Caso de las Semillas Transgénicas y su Difusión en la Argentina. In: SEMINARIO IBERO LATINOAMERICANO DE GESTIÓN TECNOLÓGICA, 11., 2005, Brasil. ALTEC - 2005. Brasil, 2005.

URRACA-RUIZ, A.; LAGUNA, E. Winning or losing a run: the role of technological drivers at stages of development. Atlantic Review of Economics, n. Special Issue: III ISEMSASE Meeting, 2018.

WOOLDRIDGE, J. Simple solutions to the initial conditions problem in dynamic, nonlinear panel data models with unobserved heterogeneity. Journal of Applied Econometrics, v. 20, n. 1, p. 39-54, 2005. 